\title{
Complex Processing of Pulverized Fly Ash by Dry Separation Methods
}

\author{
Vladimir Vasilievich Zyryanov ${ }^{1}$, Dmitry Vladimirovich Zyryanov ${ }^{2}$ \\ ${ }^{1}$ Institute of Solid State Chemistry and Mechanochemistry, Russian Academy of Sciences, Siberian Branch, Novosibirsk, Russia; \\ ${ }^{2}$ Nanopowder Technology Ltd, Novosibirsk, Russia. \\ Email: vladinetta@gmail.com,info@nanopowder-technology.com
}

Received May $18^{\text {th }}$, 2010; revised June $16^{\text {th }}$, 2010; accepted June $19^{\text {th }}, 2010$.

\begin{abstract}
Pulverized fly ash (PFA) is produced about 500 billions tons every year in the world in a result of coals combustion. Most of the fly ash collected in power plants is disposed by deposition in landfills, situated as a rule near big cities with well developed infrastructure and high cost of land. Moreover, the pollution of environmental by fine solid wastes is inevitable and takes place in area of residing of a basic part of the population. The only solution is a complex processing of fine wastes with a production of value added materials. New conception of complex processing of PFA is proposed on the base of facilities of Electro-mass-classifier (EMC) and other techniques. The characterization of separated fractions was carried out by SEM and optic microscopy, XRD, laser diffraction, Mössbauer spectroscopy and other methods. A fine fraction of glass microspheres presents the main interest as filler in various materials.
\end{abstract}

Keywords: Pulverized Fly Ash (PFA), Processing, Solid Wastes, Utilization, Dry Separation, Fly Ash Components, Glass Microspheres, Magnetospheres, Fillers, Electro-Mass-Classifier

\section{Introduction}

Coal fly ash is the particulate matter remaining after combustion of the carbonaceous component of coal. The residual, accessory minerals, predominantly clay, siliceous and iron minerals generally comprise the bulk of the ash. Fly ash was considered to be the sixth most abandoned mineral in the USA [1]. Usually less than $20 \%$ is reused commercially all over the world, predominantly in cement or as a fill material in construction [2]. The main cause of low utilization of PFA is a polydisperse inhomogeneous mix containing remnants of unburnt carbon-coke or char. Other problem for processing of PFA is related to high fineness of this waste complicating the separation of carbon by dry physical methods. Such problem may be solved by the use a green engineering-Electro-mass-classifier (EMC) technique operating with charged aerosols in closed volume in a wide range of particle sizes. Acid fly ash of type F according to ASTM prevails among different coal combustion products. In addition, commercial potential of this PFA after separation is maximal [2]. So, the perspectives of PFA complex processing technology based on the dry separation methods for environmental protection and profitable production of value added materials are better to show in such class of waste. The possible solutions for utilization with profit for other types of PFA-fly ash C according to ASTM classification and ash from low temperature boilers with fluidized bed were found as well. Based on the mechanical activation in EMC, these solutions are presented in our book [2].

\section{Materials and Methods}

Various specimens of PFA were used for investigations produced in the largest power plants with high temperature boilers of the former USSR - Reftinskaya GRES (specimen No 1, coal from Ekibastuz deposit), Troitzkaya TEC (specimen No 2, coals from Ekibastuz and Kuznetsk deposits, both plants from Ural region), Novosibirskaya TEC 5 (specimen No 3, coal from Kuznetsk deposit, Siberian region). The fineness of PFA specimens was close one to another due to use of similar electrostatic precipitators. Ash particles were ranged from 200 $\mathrm{nm}$ to $500 \mu \mathrm{m}$ with mean particles size about $40 \mu \mathrm{m}$.

Different techniques were used for dry physical separation of PFA including Electro-mass-classifier (EMC) [3,4], magnetic separator with permanent magnet from 
FeNdB-alloy (MS), triboadhesion separator (TAS), electrostatic separator with electric field $6 \mathrm{kV} / \mathrm{cm}$ (ES) and their combinations. All these devices are able to operate in discrete and continuous modes that are important for laboratory studies and industrial processing respectively. Moreover, all these installations are compatible and can operate in closed volume without aerosol contamination of air atmosphere. Flow sheet for processing of PFA may be realized for one run in production module with capacity $0.5-1 \mathrm{TPH}$. Operating in the range of particles size $\sim 50 \mathrm{~nm}-2 \mathrm{~mm}$, EMC technique allows to use with high efficiency other more selective separators after removing from PFA of the dust with particles size $<20 \mu \mathrm{m}$. Principle of operation of multifunctional green EMC engineering is based on the generation of charged aerosols and their separation to unlimited number of fractions under action of centrifugal and other forth's [3,4].

The combination of instrumental and chemical approaches was used for characterization of separated ash fractions: XRD (DRON-3M, Russia, Bruker D8 Advance,
Germany, $\mathrm{Cu} \mathrm{K} \alpha$ radiation), SEM + EDS (Hitachi TM-1000, JSM-6700F), optical microscopy Neophot 21, Germany, particle size analyzer (Laska, Russia), based on a laser diffraction method, Mössbauer spectroscopy (NZ-640, Hungary). Losses on ignition (LOI) were determined at $850^{\circ} \mathrm{C}, 1$ hour. Chemical compositions were determined in spectrometer ARL-9900XP, Swiss.

\section{Separation}

In a result of investigation of dozen various PFA specimens of $\mathrm{F}$ type, the optimal scheme for complex processing was found, Figure 1 [2]. After removing of fine and partly medium fractions in EMC, the rest of PFA can be divided by custom separators. Additionally four fractions can be obtained [2]. From practical point of view, fractions were called as potential products: Super-alpha (fine fraction No 1 in Figure 1), Alpha (medium fraction, No 2 + No 3), Delta (large fraction, No 4), coarse byproduct (No 5), magnetic byproduct (magnetic fraction, No 6). Particle size distributions for obtained products

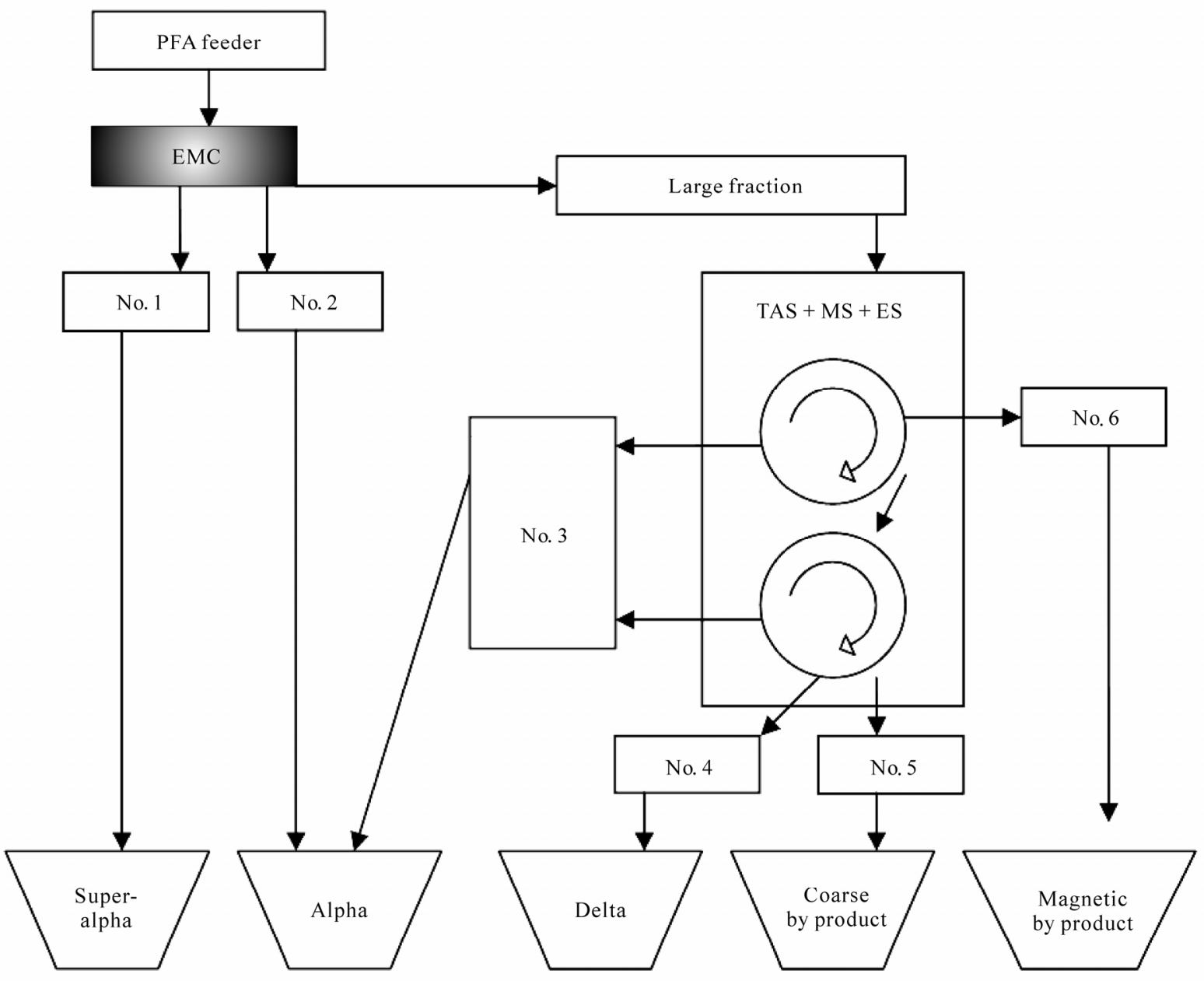

Figure 1. Flow sheet for complex dry processing of $F$ type PFA. 
are shown in Figure 2 for specimen No 1 (No 2 and 3 are very similar). Magnetic fraction has a particles size distribution close to Delta. After refinement of magnetic byproduct, magnetospheres with promising catalytic properties in methane conversion process can be received [5]. Magnetospheres present spherical particles of nanocomposites consisted from spinel and hematite in iron-rich silica glass matrix [5,6]. Coarse byproduct is a concentrate of relatively expensive materials - coke and cenospheres (hollow glass microspheres with density $<1$ $\mathrm{g} / \mathrm{cm}^{3}$ ). Dry physical methods are not efficient for more deep separation of coarse fraction. So, the yield of this byproduct must be minimized to decrease the role of wet technologies in complete processing of PFA [2]. Fractions No 2 and 3 have close fineness and properties. So, for simplification of flow sheet these fractions are reasonable for uniting in a product Alpha. The yields of products and their LOI are presented in Table 1. For enrichment of coarse fraction by coke, relatively low electric field with $\mathrm{E}=6 \mathrm{kV} / \mathrm{cm}$ was applied in ES.

The carbon content in fine fraction hardly is higher than in initial PFA due to milling of porous char particles (Figure 3) during treatment in EMC in discrete mode of operation. The decrease of velocity of rotor rotation in EMC results to lowering of LOI in fine fraction. In EMC with continuous mode of operation, the life time of ash particles in camera of charged aerosol generation sharply drops resulting to a little milling of coke and lowered contents of carbon in fine and medium fractions-Superalpha and Alpha respectively.

The ratio of milled carbon particles and capacity of EMC installations in separation process are linked parameters. From the other side, the pozzolana properties of Super-alpha product don't depend on the LOI. Moreover, after coke combustion at $850^{\circ} \mathrm{C}$, activity of Superalpha even decreases a little. There is a simple explanation of such effects. Fine carbon particles with a size $\sim n$ $\mu \mathrm{m}$ after milling get in pore space of cement stone with a little influence on the strength. In quenched ash glass particles mechanical strains results to high chemical activity in basic solution in mortar (so called mechanochemical activity). After thermal annealing, chemical activity decreases due to relaxation of mechanical strains in glass particles. Weak effects of carbon and strain relaxation on the strength of mortar with additives of Super-alpha show counterly signs and compensate each other.

\section{Chemical Composition}

Chemical compositions of obtained products are presented in Table 2. These results are very similar to other known data [2]. The iron content in Delta is lowered

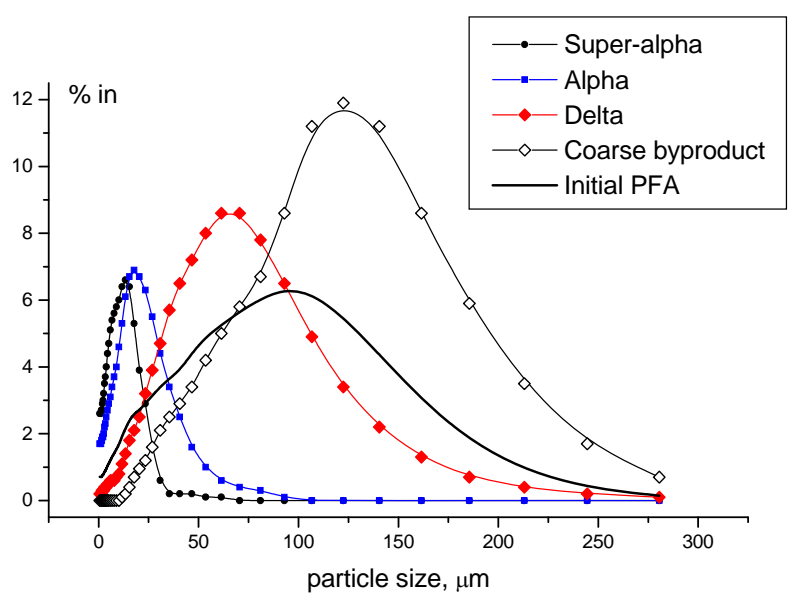

Figure 2. Particle size distributions for obtained products from PFA No 1.

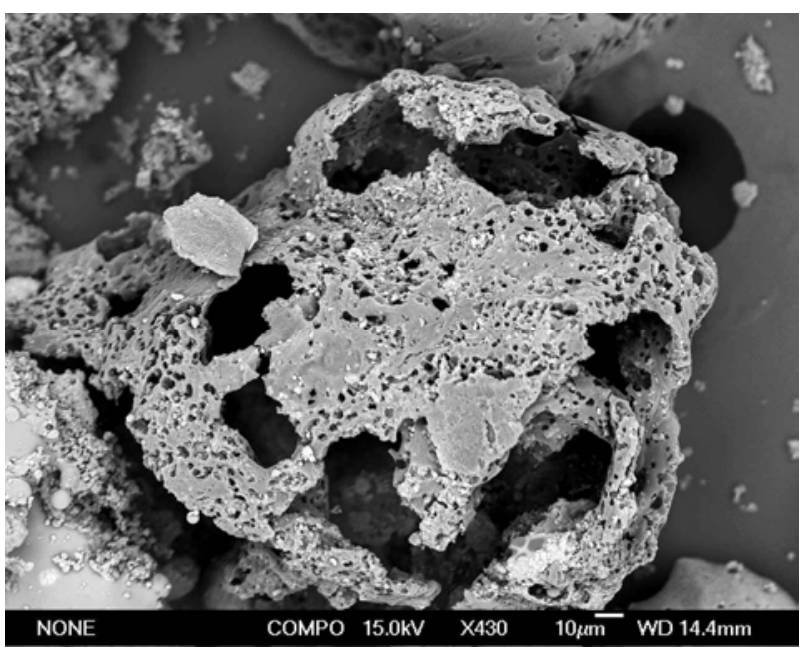

Figure 3. Porous coke particle from PFA No 1.

relatively Alpha due to magnetic separation of large fraction only. Nevertheless, in EMC there is a facility to remove magnetic particles from fine fractions as well, if this operation has practical sense for better commercialization.

\section{Phase Composition}

Phase compositions of all studied PFA specimens are very similar-small crystallites of mullite (m) and quartz (q) in silica glass, Figure 4. The content of mullite depends on the particle size. Fine fractions are enriched by quartz, and coarse fractions by mullite that correlates with chemical composition. Cenospheres are usually enriched by silica and $\mathrm{Na} / \mathrm{K}$, so the mullite content is lowered relatively main part of microspheres with density $>$ $1 \mathrm{~g} / \mathrm{cm}^{3}$. Phase compositions of magnetic fractions differ slightly as well, Figure 5. The main crystal phase is fer- 
Table 1. Yields and LOI (\%) of products obtained in flow sheet in Figure 1.

\begin{tabular}{|c|c|c|c|c|c|c|c|c|c|c|c|}
\hline \multirow{3}{*}{ PFA, No } & \multirow{3}{*}{ LOI } & \multicolumn{10}{|c|}{ Products } \\
\hline & & \multicolumn{2}{|c|}{ Super-alpha } & \multicolumn{2}{|c|}{ Alpha } & \multicolumn{2}{|c|}{ Delta } & \multicolumn{2}{|c|}{ Coarse byproduct } & \multicolumn{2}{|c|}{$\begin{array}{l}\text { Magnetic } \\
\text { byproduct }\end{array}$} \\
\hline & & $\mathrm{Y}$ & LOI & $\mathrm{Y}$ & LOI & $\mathrm{Y}$ & LOI & $\mathrm{Y}$ & LOI & $\mathrm{Y}$ & LOI \\
\hline 1 & 2.8 & 9 & 3.6 & 45 & 2.1 & 36 & 2.7 & 7 & 4.9 & 3 & 1.0 \\
\hline 2 & 3.5 & 8 & 4.4 & 39 & 2.4 & 39 & 3.0 & 12 & 8.4 & 2 & 1.0 \\
\hline 3 & 3.9 & 7 & 4.5 & 35 & 2.9 & 45 & 3.2 & 10 & 10.9 & 3 & 1.2 \\
\hline
\end{tabular}

Table 2. Chemical composition of obtained products (for magnetospheres after refinement).

\begin{tabular}{|c|c|c|c|c|c|c|c|c|c|c|c|}
\hline PFA & Product & $\mathrm{SiO}_{2}$ & $\mathrm{Al}_{2} \mathrm{O}_{3}$ & $\mathrm{Fe}_{2} \mathrm{O}_{3}$ & $\mathrm{CaO}$ & $\mathrm{MgO}$ & $\mathrm{TiO}_{2}$ & $\mathrm{~K}_{2} \mathrm{O}$ & $\mathrm{Na}_{2} \mathrm{O}$ & $\mathrm{P}_{2} \mathrm{O}_{5}$ & $\mathrm{MnO}$ \\
\hline \multirow{4}{*}{1} & S-alpha & 61.3 & 24.4 & 3.72 & 1.97 & 0.96 & 1.0 & 0.81 & 0.58 & 0.62 & 0.08 \\
\hline & Alpha & 60.5 & 26.1 & 5.64 & 2.39 & 1.09 & 1.18 & 0.68 & 0.39 & 0.40 & 0.11 \\
\hline & Delta & 58.9 & 29.5 & 2.18 & 2.54 & 0.99 & 1.29 & 0.61 & 0.27 & 0.41 & 0.06 \\
\hline & Magnetospheres & 26.8 & 10.9 & 55.1 & 2.35 & 1.14 & 0.91 & 0.17 & 0.43 & 0.10 & 1.93 \\
\hline \multirow{4}{*}{2} & S-alpha & 62.7 & 24.0 & 4.0 & 1.55 & 0.7 & 1.06 & 0.8 & 0.4 & 0.41 & 0.07 \\
\hline & Alpha & 60.1 & 26.1 & 5.1 & 1.74 & 0.76 & 1.23 & 0.59 & 0.36 & 0.48 & 0.14 \\
\hline & Delta & 59.2 & 28.5 & 3.5 & 1.87 & 0.69 & 1.42 & 0.45 & 0.27 & 0.44 & 0.1 \\
\hline & Magnetospheres & 24.9 & 9.69 & 57.1 & 1.66 & 1.59 & 0.95 & 0.26 & 0.34 & 0.25 & 1.95 \\
\hline \multirow{4}{*}{3} & S-alpha & 62.0 & 20.2 & 4.22 & 2.05 & 1.52 & 0.75 & 2.9 & 1.59 & 0.5 & 0.05 \\
\hline & Alpha & 59.8 & 22.3 & 4.96 & 2.82 & 1.88 & 0.86 & 2.81 & 1.52 & 0.45 & 0.10 \\
\hline & Delta & 57.9 & 23.8 & 4.15 & 3.49 & 1.69 & 0.99 & 2.67 & 1.33 & 0.36 & 0.06 \\
\hline & Magnetospheres & 22.3 & 7.1 & 61.1 & 2.91 & 2.90 & 0.10 & 0.41 & 0.79 & 0.45 & 2.4 \\
\hline
\end{tabular}

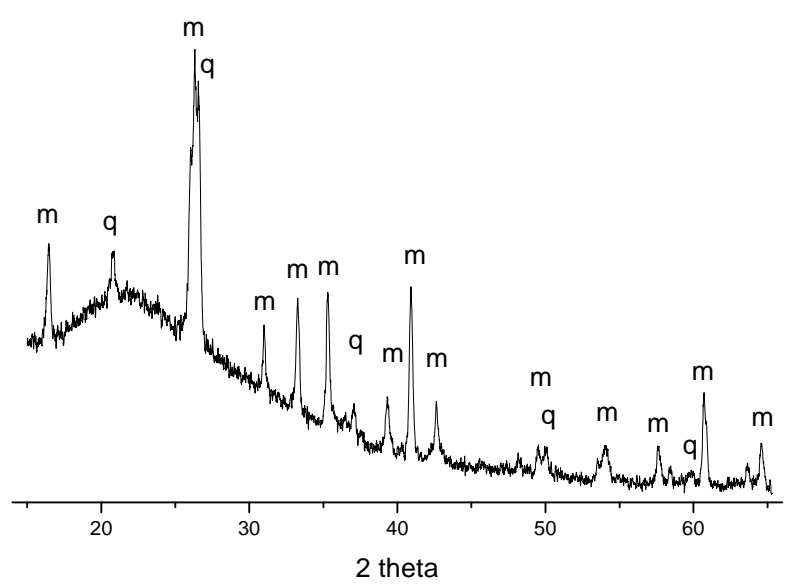

Figure 4. XRD pattern for PFA No 1.

rospinel, and minor phases are hematite (h) and quartz (q). The content of ferrospinel depends on the magnetosphere size with maximum content in medium fractions.

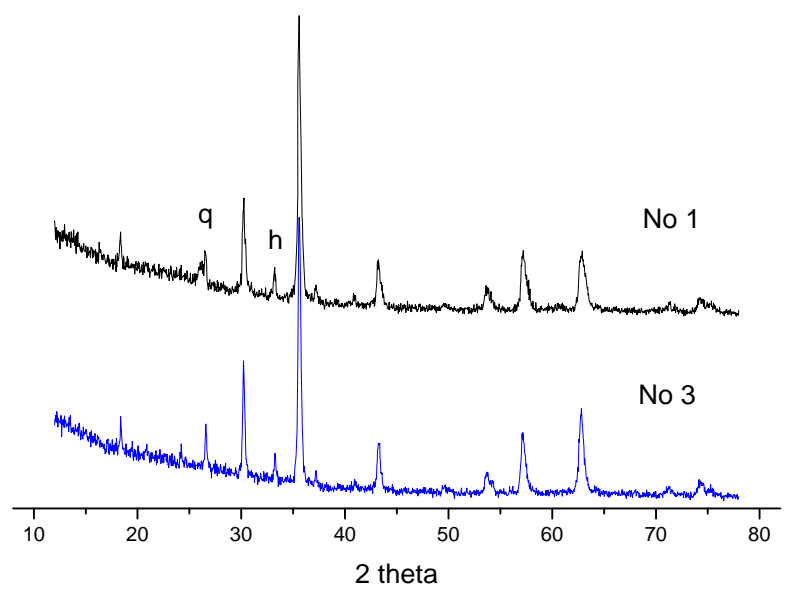

Figure 5. XRD patterns of magnetospheres.

\section{Morphology}

Morphology of the most ash particles presents hollow 
glass microspheres, Figures 6-8 (marks in $\mu \mathrm{m}$ ). In large fractions some glass particles have irregular forms. In coarse fraction most particles are presented by agglomerates, including plerospheres and composites, Figures 9-10.

For many applications, spherical form of glass particles is very attracting, especially as filler in cement, concrete mixtures, polymer composites, and so on. So, the removing from PFA of coarse byproduct, consist of coke and nonspherical glass particles with irregular form and porous microstructure sharply enhances the quality of obtained products as fillers.

Part of carbon is in aggregates with silica glass microspheres, Figure 10. Because of this, the efficient removing of coke from PFA is limited by individual coke particles only. EDX analysis of glass particles in Figure 9 displays a different chemical composition: for particle 1 -

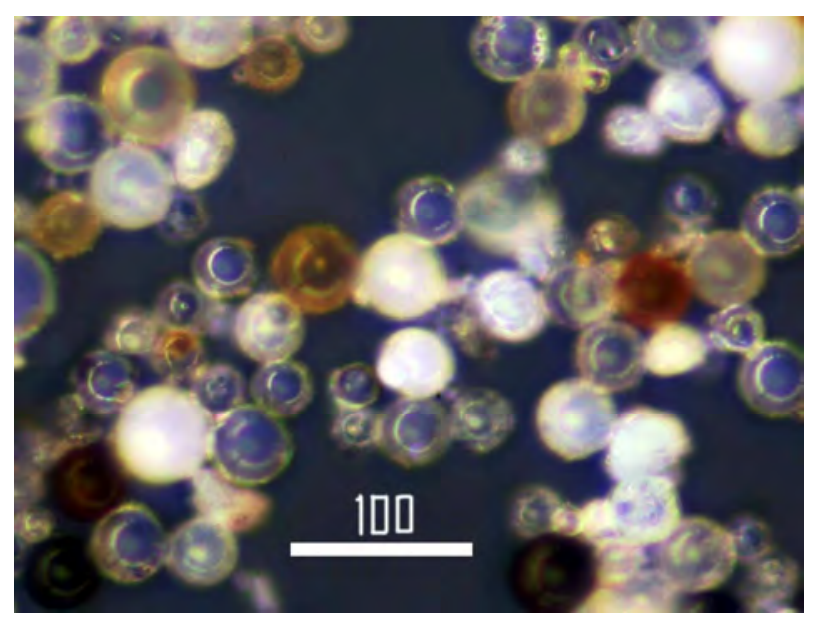

(a)

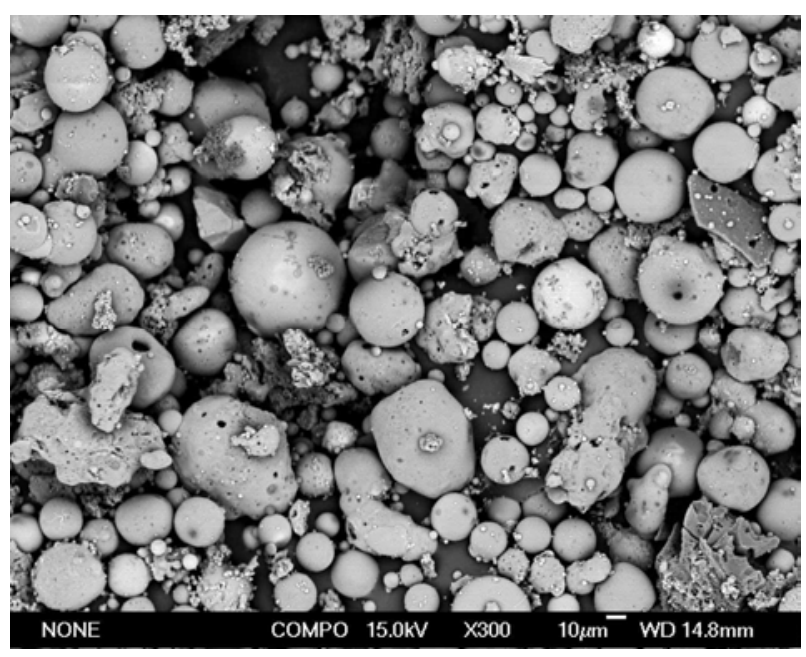

(b)

Figure 6. Product Alpha. (a) optical image (PFA No 2); (b) SEM image (PFA No 1).

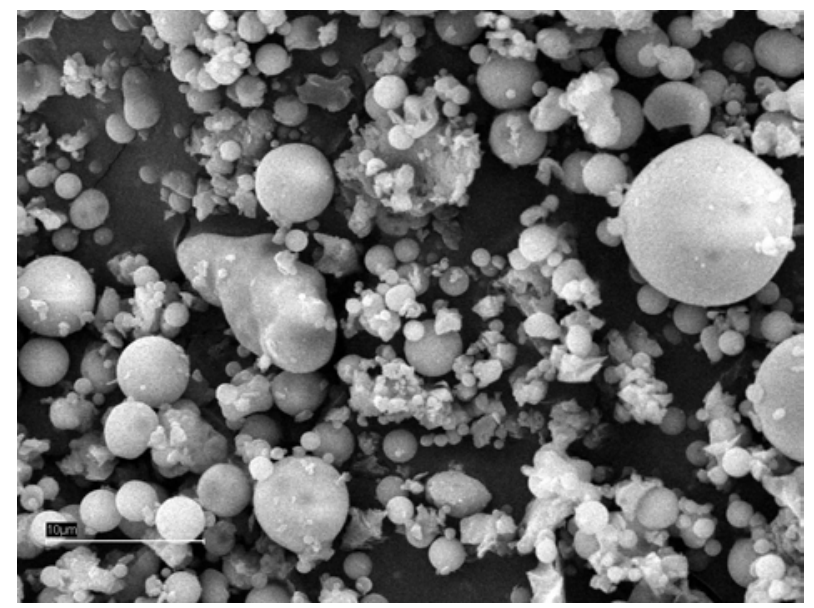

Figure 7. SEM image of Super-alpha No 1.

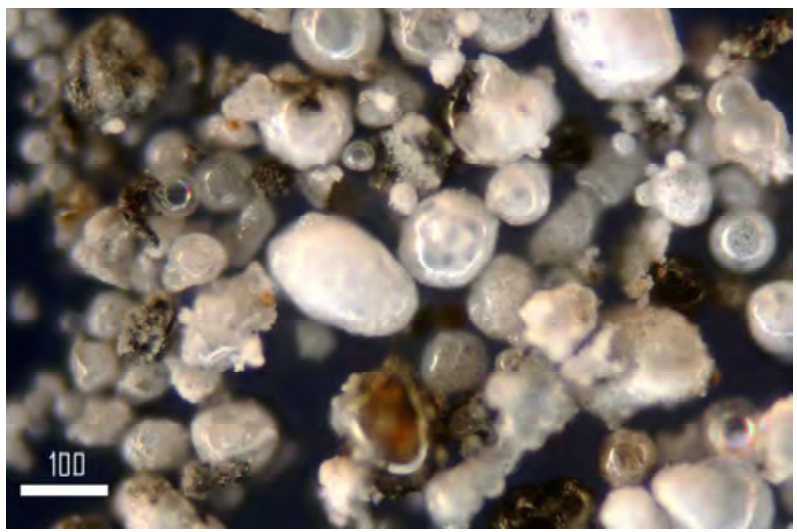

Figure 8. Optical image of Delta No 2.

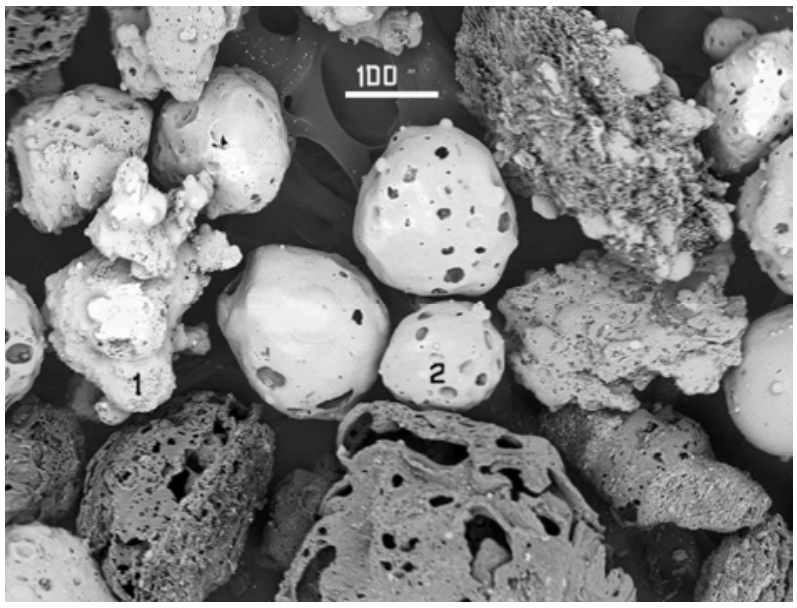

Figure 9. SEM image of coarse byproduct No 1.

$\mathrm{Si} / \mathrm{Al}=1.1$, for particle $2-\mathrm{Si} / \mathrm{Al}=2.1$, dark particles coke aggregates.

Magnetospheres display various morphological types in every kinds of PFA, Figures 11-13. Magnetospheres from different power plants were studied by combination 


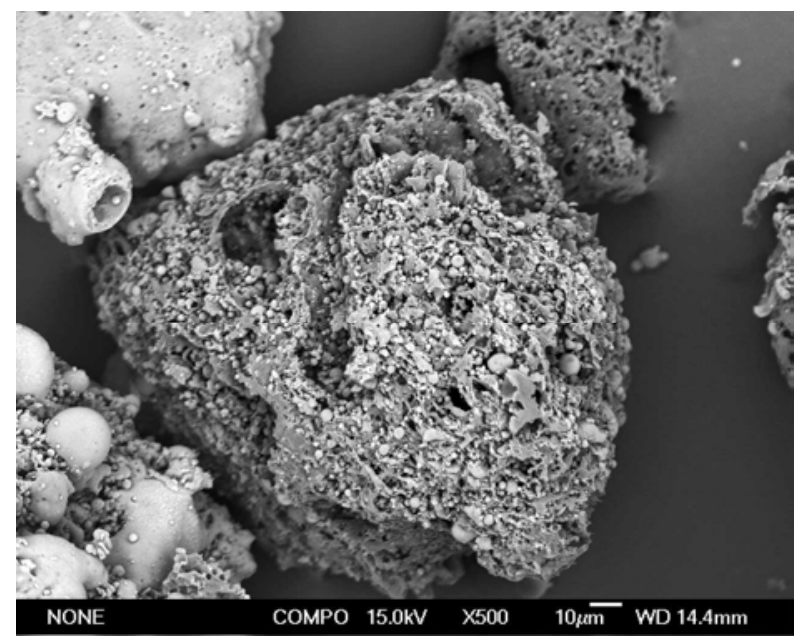

Figure 10. Aggregate from coke and glass microspheres in coarse byproduct No 1.

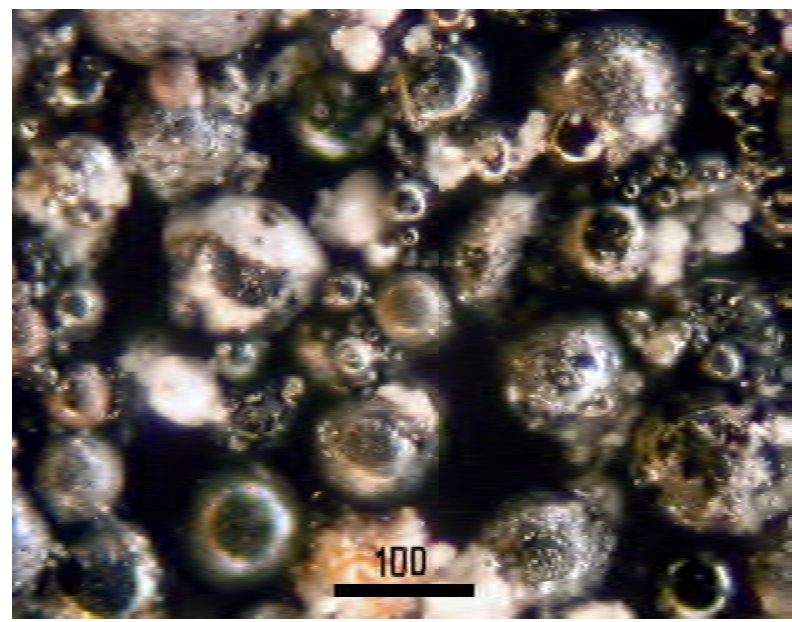

(a)

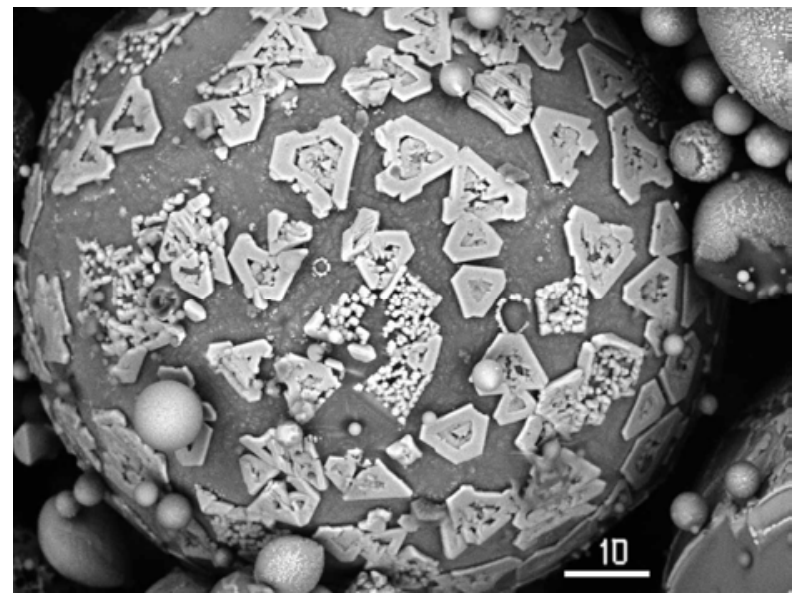

(b)

Figure 11. Optical (a) and SEM (b) images of magnetospheres No 1.

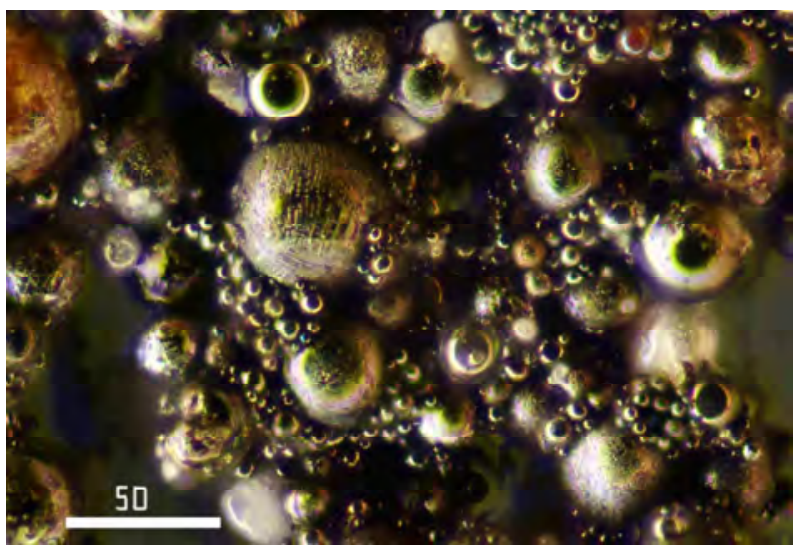

(a)

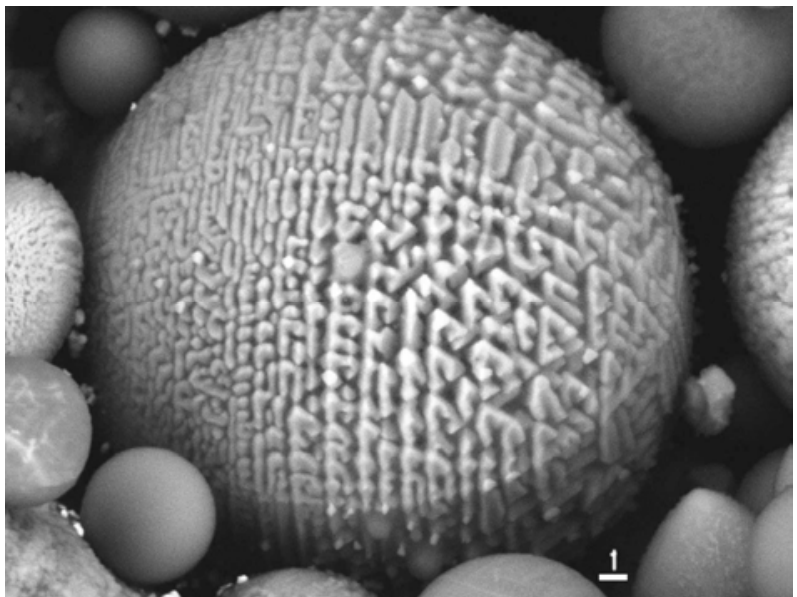

(b)

Figure 12. Optical (a) and SEM (b) images of magnetospheres No 2.

of structural methods-XRD with Rietveld analysis and Mössbauer spectroscopy [6]. Structural formulas of ferrospinels were determined that allows to search a correlations with catalytic properties of magnetospheres with different origin [5]. Such correlations help to select optimal magnetospheres and modify them for promising applications in catalysis.

Cenospheres separated from coarse byproducts by wet technology are shown in Figure 14. Cenospheres are well known product with a relatively high cost and large area for applications [2]. The content of cenospheres in coarse byproduct is about $2-4 \%$ (up to $30 \%$ of total content in PFA).

The iron contents in nonmagnetic fractions of glass microspheres and cenospheres in studied specimens of PFA are in the range $3-5 \%$. As a result, the color of glass microspheres usually grey, that complicates their applications in white and colored polymer compositions. According to studies of glasses by Mössbauer spectroscopy 


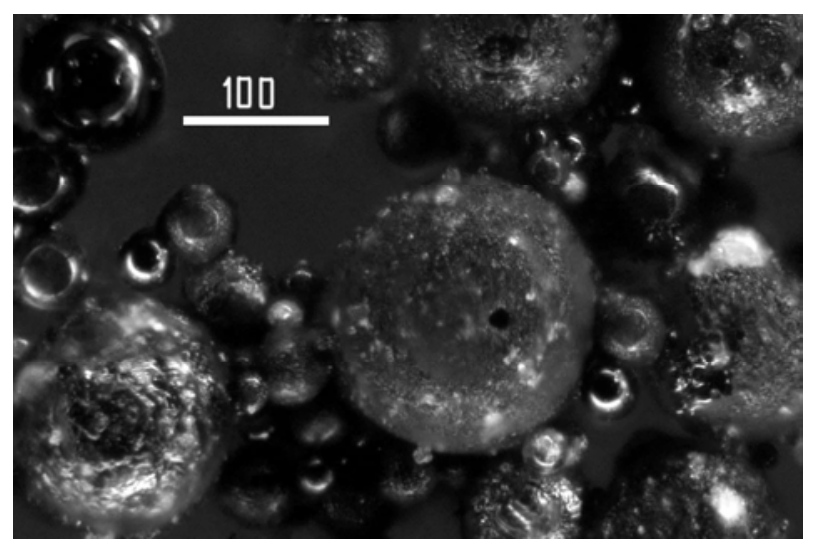

(a)

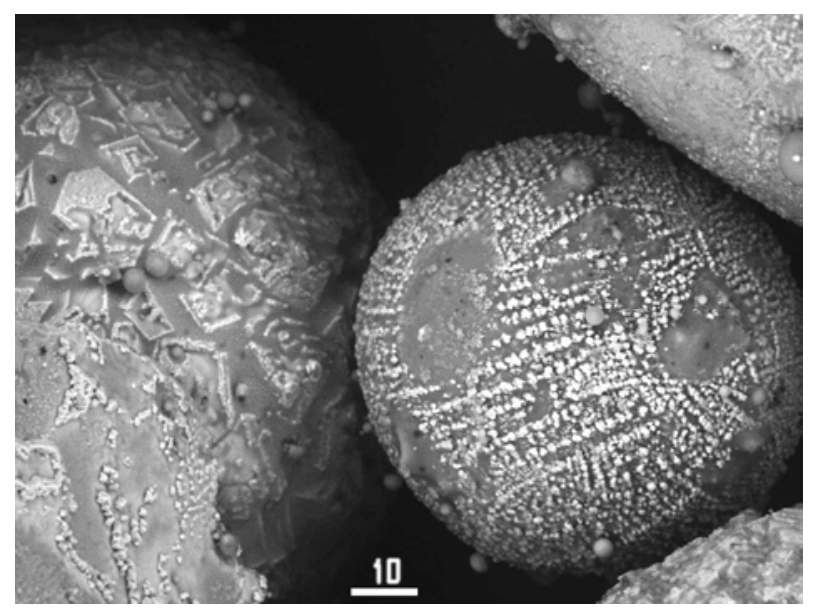

(b)

Figure 13. Optical (a) and SEM (b) images of magnetospheres No 3.

[2], $\mathrm{Fe}^{2+}$ state have coordination number 4.5 and $\mathrm{Fe}^{3+}-6$ (usual octahedral position). Parameters of iron in glass are presented in Table 3. After thermal treatment in air, the content of $\mathrm{Fe}^{2+}$ decreases with narrowing of lines that corresponds to annealing of mechanical strains in quenched glass. The color of powders acquires poorly red shade.

Other valuable product which can be separated from coarse byproduct is a coke with LOI $>90 \%$. The content of extracted coke in coarse byproducts was about 2-4\%. A coke represents a practical interest not only as a clean solid fuel, but mostly a source of rare volatile elementsV, In, Co, U (Figure 16), and as absorbent [2].

The obtaining of cenospheres and coke makes possible a profitable wet chemical processing of coarse byproduct. In such a case the waste after separation coke and cenospheres presents the source of rare earth elements, secondary products are silica, alumina and raw for zeolite synthesis [2]. Coarse byproduct may be used without

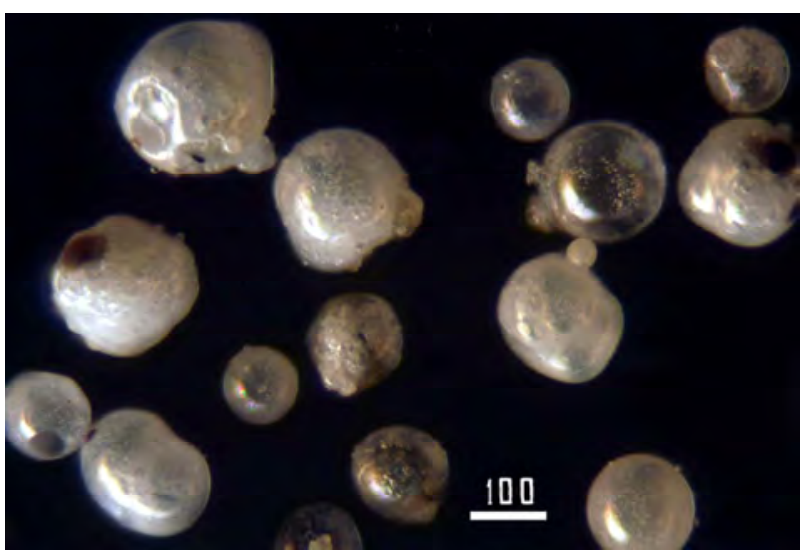

(a)

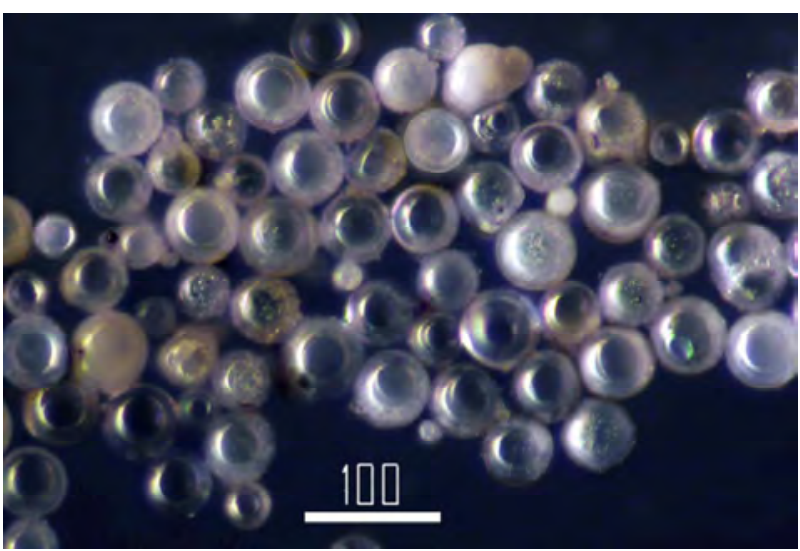

(b)

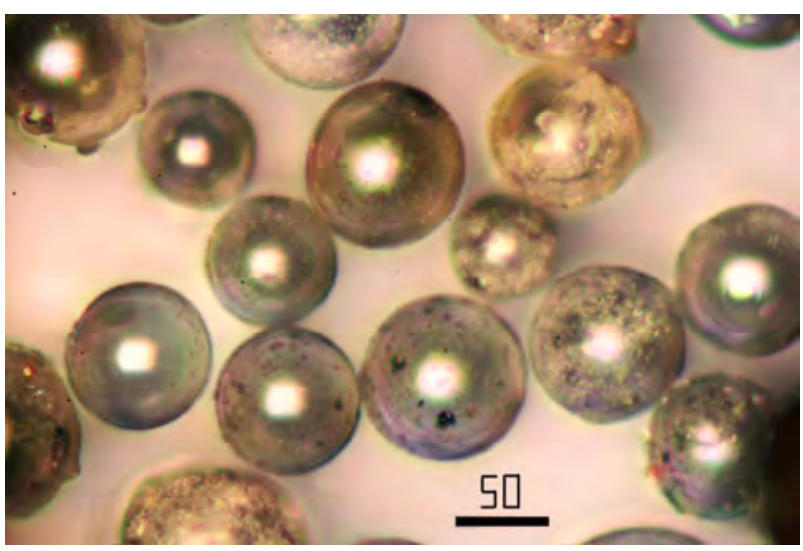

(c)

Figure 14. Optical images of cenospheres divided from PFA No 1 (a), 2 (b) and 3 (c).

processing in fabrication of low dense building ceramics. However, the capacity of region market usually is less than quantity of produced coarse byproduct. Diversification of value added products allows to realize with profit a complete utilization of produced PFA. 
Table 3. Parameters of Mössbauer spectra of nonmagnetic cenospheres separated from PFA No 3.

\begin{tabular}{|c|c|c|c|c|c|c|c|c|}
\hline \multirow{2}{*}{ parameters } & $\Gamma_{1}$ & $\delta_{1}$ & $\varepsilon_{1}$ & content, \% & $\Gamma_{2}$ & $\delta_{2}$ & $\varepsilon_{2}$ & content, \% \\
\hline & \multicolumn{4}{|c|}{ Dublet $\mathrm{Fe}^{3+}$} & \multicolumn{4}{|c|}{ Dublet $\mathrm{Fe}^{2+}$} \\
\hline initial & 0.868 & 0.333 & 1.154 & 61 & 0.778 & 1.010 & 1.945 & 39 \\
\hline $\mathrm{T}=850^{\circ} \mathrm{C}$ & 0.838 & 0.322 & 0.991 & 88 & 0.560 & 1.007 & 2.088 & 12 \\
\hline
\end{tabular}

$\Gamma$ - width of lines, $\delta$ - chemical shift, $\varepsilon$ - quadruplet splitting.

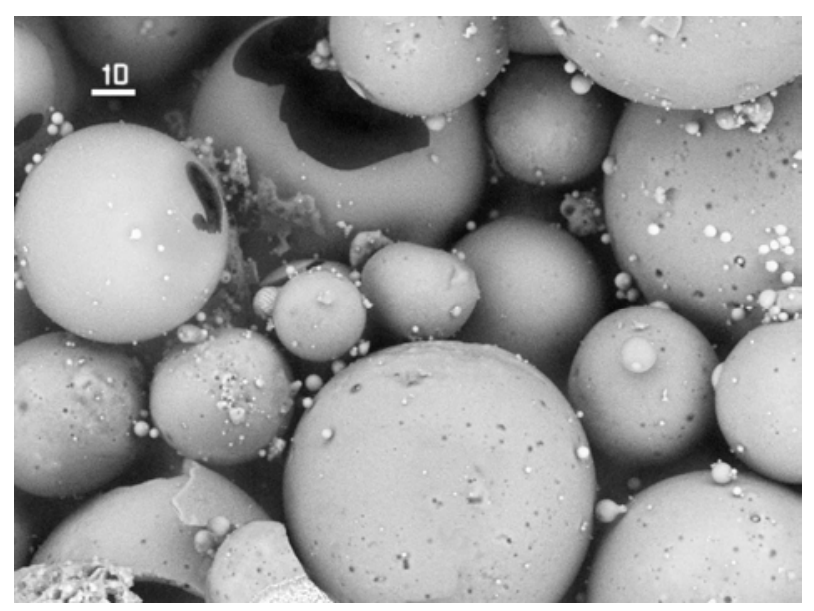

Figure 15. Typical SEM images of cenospheres obtained from PFA No 2.

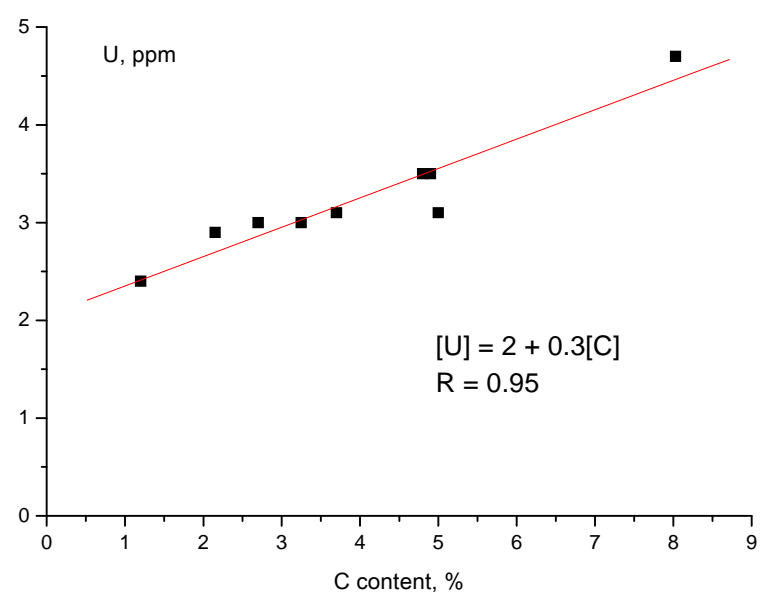

Figure 16. Correlation between $U$ and carbon contents in fractions of PFA No 1.

\section{Pozzolana Properties}

The utilization of PFA products is mostly as fillers in cement and concrete. The profitable processing of PFA is determined by the capacity of construction market. The quality of PFA products determines the possible volume of utilization. The pozzolana properties are shown in Figure 17 for the fine PFA products.

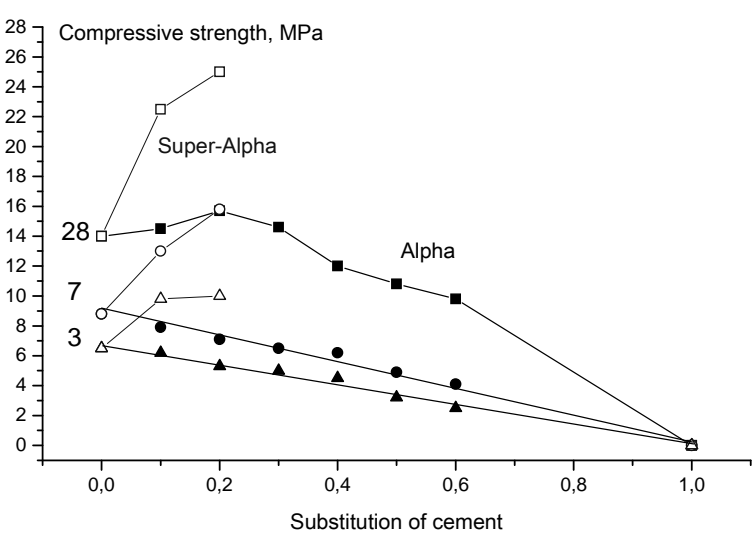

Figure 17. Compressive strength for mortars cement:sand = 1:3 with substitution of cement by products from PFA No 1 after 3, 7 and 28 days of curing in wet atmosphere.

Partial substitution of cement by Super-alpha drastically increases the strength of mortars even after short time of curing. This enhancement of building material is related to increasing of density of concrete due to filling of pores between cement particles and absorption of excessive $\mathrm{Ca}(\mathrm{OH})_{2}$. The substitution of $30 \%$ cement by Alpha results to the same strength properties of concrete. The fineness of Alpha and custom cement are very similar, so the effect of substitution is related to pozzolana properties. A possible use of Delta in mortars or ready mix is due to partial substitution of sand. In this case a workability of mortars becomes better thanks to spherical form of particles, but the compressive strength displays a weak dependence on the degree of substitution. However, the density of concrete decreases, so normalized on the density effect becomes significant, Table 4 [2]. The possible volume of PFA utilization in construction industry becomes much more significant after processing. Relatively low quality of mortars with initial PFA is related to remnants of coke and porous coarse ash particles with irregular form.

In the case of specially designed coal mix with inorganic additives, prepared with optimal morphology, the cost of PFA after combustion becomes more significant than produced electric and heat energy [7]. In other words, electric and heat energy must become byproduct 
Table 4. Parameters of 28 day concretes with products separated from PFA No 1, which are equal on the volume to mix cement:sand = 1:2 (weight ratio).

\begin{tabular}{|c|c|c|c|c|}
\hline No & Composition & Density $\rho, \mathrm{g} / \mathrm{cm}^{3}$ & $\begin{array}{c}\text { Compressive strength } \sigma \text {, } \\
\mathrm{MPa}\end{array}$ & Normalized strength, $\sigma / \rho$ \\
\hline 1 & Cement: 2sand & 2.43 & $36(3)$ & 14.8 \\
\hline 2 & Cement: sand + Delta & 2.09 & $32(3)$ & 15.3 \\
\hline 3 & Cement: 2Delta & 1.64 & $22(2)$ & 13.4 \\
\hline 4 & Cement: 2magnetospheres & 2.095 & $34.5(1.5)$ & 16.5 \\
\hline 5 & (2/3cement + 1/3Alpha): 2sand & 2.37 & $32(1.5)$ & 13.5 \\
\hline 6 & (2/3cement + 1/3Alpha): sand + Delta & 2.08 & $30(3)$ & 14.4 \\
\hline 7 & (2/3cement + 1/3PFA): sand + PFA & 2.09 & $24(1)$ & 11.5 \\
\hline
\end{tabular}

only in chemical reactors for the production of high temperature materials-glass microspheres and other fillers, binding materials, and so on.

\section{Conclusions}

PFA from coal power plants consist of components with a number of promising applications. One run dry physical separation in green EMC-based installation of PFA to 3 products and 2 byproducts provides wide possibilities for utilization with profit of solid wastes. Refinement of magnetic byproduct and wet processing of coarse byproduct can provide complete processing of solid wastes to value added products. In such a case, the effect of elimination environmental pollution is accompanied by economy of natural resources and lowering of $\mathrm{CO}_{2}$ emission. Obtained results promotes to new eco-friendly conception to produce a power as a byproduct in relatively small chemical reactors which can provide better economic perspectives due to local green production of distributed energy from carbonaceous sources.

\section{Acknowledgements}

The first author gratefully acknowledges the support of this research in part through grant Russian Found for Basic Researches 09-03-00364.

\section{REFERENCES}

[1] G. L. Fisher, "Biomedically Relevant Chemical and Physical Properties of Coal Combustion Products,” Environmental Health Perspectives, Vol. 47, 1983, pp. 189-199.

[2] V. V. Zyryanov and D. V. Zyryanov, "Fly Ash—Technogenic Raw,” Maska Ltd, Moscow, 2009, p. 320.

[3] V. V. Zyryanov, “Electro-Mass-Classifier,” USSR Invention Certificate No 1403439, 1988.

[4] V. V. Zyryanov, D. V. Zyryanov and V. A. Sadykov, "Creation of Coatings by Charged Aerosol Deposition Method,” Russian Nanotechnologies, Vol. 3, No. 5-6, 2008, pp. 118-126.

[5] A. G. Anshits, E. V. Kondratenko, E. V. Fomenko, et al., "Novel Glass Crystal Catalysts for the Processes of Methane Oxidation,” Catalysis Today, Vol. 64, No. 1-2, 2001, pp. 59-67.

[6] V. V. Zyryanov, S. P. Petrov and A. A. Matvienko, "Morphology and Structure of Magnetic Spheres Based on Hematite or Spinel and Glass," Inorganic Materials, Vol. 46, No. 6, 2010, pp. 651-659.

[7] V. V. Zyryanov and D. V. Zyryanov, "Chemical Reactors as Alternative to Coal Burning Boilers in Power Plants," Proceedings of 7th All-Russian Conference with International Participation "Combustion of solid fuel”, Vol. 3, 10-13 November 2009, Novosibirsk, pp. 19-25. 